Citation/Refer Denys S, Hofmann M, van Wieringen A, Wouters J

ence

Improving the efficiency of the Digit Triplet Test using digit scoring with variable adaptive step sizes

Int. J. Audiol, 1-8, Jun 2019

Archived

Author manuscript: the content is identical to the content of the published

version paper, but without the final typesetting by the publisher

Published https://www.tandfonline.com/doi/abs/10.1080/14992027.2019.1622042?jo

version

urnalCode=iija20

DOI

$10.1080 / 14992027.2019 .1622042$

Journal

https://www.tandfonline.com/action/journallnformation?show=aimsScope\&j

homepage

ournalCode=IIJA20

Author

sam.denys@kuleuven.be, jan.wouters@med.kuleuven.be

contact 
2 Improving the efficiency of the Digit Triplet Test using digit scoring with

3 variable adaptive step sizes

4 Sam Denys*-Michael Hofmann, Astrid van Wieringen and Jan Wouters

5 KU Leuven, Department of Neurosciences, ExpORL, Leuven, Belgium

$6 *$ Herestraat 49 - box 721, 3000 Leuven, Belgium, sam.denys@kuleuven.be 
7 Acknowledgements: The authors thank Erica Schoonjans, Babette Van De Velde and Lien 8 Zwysen (master students of the Speech-Language Pathology and Audiology program, KU 9 Leuven) for their help with participant recruitment and data collection. Heleen Luts is 10 acknowledged for her methodological support.

11 Portions of this article were presented at the European Federation of Audiology (EFAS) 12 conference (Istanbul, Turkey, May 27-30, 2015).

13 This work was supported by a TBM-FWO grant from the Research Foundation Flanders (grant 14 number T002216N) and by KU Leuven project OT/12/98.

15 The authors report no conflicts of interest. 


\section{Improving the efficiency of the Digit Triplet Test using digit scoring with \\ 17 variable adaptive step sizes}

Objective: To investigate modifications of the Flemish Digit Triplet Test (DTT) to improve its efficiency, i.e. the use of a low-pass filtered noise and variable adaptive step sizes according to a digit scoring procedure, targeting different recognition probabilities. Design and study sample: Speech reception thresholds (SRT) were evaluated in terms of their test-retest reliability and correlation with pure tone averages (PTA) in a group of 68 adult participants with different degrees of hearing impairment. Results: The use of a low-pass filtered noise did not result in better test-retest reliability or an improved SRT-PTA correlation. Using digit scoring with adaptive step sizes parametrized to target a recognition probability of $79 \%$ (D79), corresponding to the recognition probability of the currently used DTT with triplet scoring and fixed adaptive step sizes of $2 \mathrm{~dB}$, increased test-retest reliability. Lower recognition probabilities of 57 and $35 \%$ demonstrated worse reliability and worse SRT-PTA correlations. Conclusions: Given the increased test-retest reliability of D79, a similar reliability as for the currently used DTT could be obtained after considerably fewer trials, leading to a profit in test duration.

Keywords: Digit Triplet Test; efficiency; hearing screening 
Digit Triplet Tests (DTT) are valid, reliable and robust speech-in-noise tests for the detection of hearing impairment. They use a limited set of highly familiar speech items, i.e. three-digit combinations, which are presented in a noise masker to estimate an individual's speech reception threshold (SRT), i.e. the signal-to-noise ratio (SNR) that is required for a certain recognition probability. For this purpose, an adaptive procedure in which the SNR is varied per triplet is used and an averaged SNR over $n$-presentation levels or trials is calculated.

Today, fifteen years after their original conception (Smits et al, 2004), DTTs are available in multiple languages (Zokoll et al, 2012; Zokoll et al, 2013; Potgieter et al, 2015; Willberg et al, 2016) for large-scale hearing screening over telephone (Smits \& Houtgast, 2005; Jansen et al, 2010; Watson et al, 2012), the internet (Smits et al, 2006; Vlaming et al, 2014) or via mobile devices, such as smartphones (Potgieter et al, 2018a; De Sousa et al, 2018) and tablet computers (Denys et al, 2018). Also, their development principles have been consolidated in a consensus paper by the International Collegium of Rehabilitative Audiology (Akeroyd et al, 2015).

DTT-SRTs agree strongly with pure tone averages (PTA) derived from diagnostic pure tone audiometry, with reported correlations between 0.7 and 0.8 , depending on PTA definitions and study group characteristics (Smits et al, 2004; Jansen et al, 2010; Watson et al, 2012; Koole et al, 2016; Potgieter et al, 2018b). As a consequence, high sensitivity and specificity values of $>80 \%$ to detect hearing impairment have been obtained when appropriate pass/fail criteria are used (Smits et al, 2004; Watson et al, 2012; Jansen et al, 2013; Vlaming et al, 2014; Koole et al, 2016; Folmer et al, 2017; Potgieter et al, 2018a;

Vercammen et al, 2018; Potgieter et al, 2018b). Their feasibility as an automatic self-test, robustness to ambient noise levels outside of audiometric booth environments, and low 
linguistic (Warzybok et al, 2015; Kaandorp et al, 2016) and cognitive demands have

60 contributed to widespread use for hearing screening.

In adults, the test takes about 3 to 4 minutes to perform. Next to aiming at the shortest test duration possible, achieving high reliability or a low measurement error is important with respect to screening test efficiency. More precisely, the ratio between the standard deviation of SRTs between participants and the measurement error should be as high as possible.

Efforts have been made by researchers to improve the efficiency of existing DTTs by experimenting with test modifications that would result in a larger spread of SRTs among participants. Especially the use of alternative maskers has been explored to sensitize the test for the detection of (high-frequency) hearing impairment (after Leensen et al, 2011): e.g. using fluctuating (Smits \& Houtgast, 2007) or low-pass filtered (Vlaming et al, 2014; Vercammen et al, 2018) rather than continuous broadband speech-shaped noise that is spectrally identical to the speech material.

These modifications have improved the correlation between SRTs and PTAs, and because of their improved sensitivity for high-frequency hearing impairment, speech-innoise screening tests using low-pass filtered rather than broadband noise are being commonly used, e.g. for the detection of noise-induced hearing impairment in the framework of occupational healthcare (Leensen \& Dreschler, 2013; Sheikh Rashid et al, 2017; Jansen et al, 2014), but also targeting adolescents for whom concerns regarding recreational noise-induced hearing impairment are growing (Rashid et al, 2016). Inconsistent findings have been published with respect to the reliability of such tests, with some suggesting no impact of using low-pass filtered noise on test reliability (Leensen et al, 2011), and some suggesting a decrease in reliability (Jansen et al, 2014; Vlaming et al, 2014). 
When properly homogenized speech materials are used, test-retest reliability for currently available DTTs is well below $1 \mathrm{~dB}$, reflecting a steeply sloped psychometric curve. This is relevant for emerging longitudinal hearing research using DTTs (Pronk et al, 2013; Pronk et al, 2014; Stam et al, 2015; Stam et al, 2016), as well as for hearing screening programs that intend to follow-up on hearing over time (Denys et al, 2018). Less research has been devoted to increasing DTTs' test-retest reliability to improve test efficiency. Hence, this study aimed at investigating test modifications that might increase test-retest reliability. In particular, the use of variable adaptive step sizes according to a digit scoring method was explored. Usually, the presentation level is adapted per trial with a fixed step size of $2 \mathrm{~dB}$ based on the response for a complete triplet; i.e. the SNR is decreased in case of a correctly identified triplet (the test becomes more difficult), and increased in case of an incorrect triplet response (the test becomes easier). For matrix tests, presenting syntactically fixed but semantically unpredictable sentences in noise constructed from an underlying word matrix of ten names, verbs, numerals, adjectives and nouns, adaptive step sizes that depend on the number of correctly identified words per trial are used, and this has resulted in an improved reliability of the obtained

100 SRTs (Brand \& Kollmeier, 2002). This principle might also be applicable to DTTs if the step size is varied according to the number of individual digits per triplet that are recognized correctly.

Generally, the probability to recognize a digit correctly $p$, i.e. the recognition

104 probability, is linked to the steps $S_{i}$ (in $\mathrm{dB}$ ) for $i$ correctly recognized digits by the 105 weighted sum of the probability mass function of the binomial distribution

$$
\sum_{i=0}^{3} S_{i}\left(\begin{array}{l}
3 \\
i
\end{array}\right) p^{i}(1-p)^{3-i}=0
$$


107 For triplet scoring, using equal adaptive steps of $S=2 \mathrm{~dB}$, results in an average

108 recognition probability of approximately $79 \%$ for a single digit. Indeed, the $50 \%$

109 recognition probability for a triplet is the product of the recognition probabilities of $79 \%$

110 for individual digits $\left(p^{3}\right)$. For digit scoring, the step size depends on the number of

111 correctly recognized digits $i$. Here, the steps are linearly spaced across the same range as

112 used for triplet scoring, i.e. $2 \mathrm{~S}=4 \mathrm{~dB}$, which for a given recognition probability $p$ results

113 in a step size

$$
S_{i}=2 S\left(p-\frac{i}{3}\right)
$$

As such, by simply adjusting the adaptive step size depending on the number of

116 digits correctly identified, any recognition probability desired can be targeted, and a DTT

117 using digit scoring can be parametrized to target the same recognition probability as a

118 DTT with triplet scoring, i.e. 79\%, resulting in similar SRTs. Importantly, modifications

119 applied to the baseline DTT (i.e. the original test) should not affect its validity in terms

120 of the correlation between the SRT and the PTA. Also, in order to reach improved

121 efficiency (by increasing test-retest reliability), the spread in SRTs among individuals,

122 which can also be expressed in terms of the SRT-PTA correlation, should remain 123 unaltered.

124 As a first study aim, it was investigated whether a DTT using low-pass filtered noise would result in equal or worse test-retest reliability as compared to the baseline

126 DTT with broadband speech-shaped noise, and whether an improved SRT-PTA 127 correlation could be achieved.

128 A second aim of this study was to investigate whether using a digit scoring 129 procedure would result in increased test-retest reliability compared to the baseline DTT, 130 without affecting its correlation with the PTA. Also, an evaluation of DTT procedures 
131 targeting other recognition probabilities, i.e. $57 \%$ and $35 \%$, in comparison to the

132 commonly used recognition probability of $79 \%$, in terms of test-retest reliability and 133 agreement with the PTA was conducted. 


\section{Materials and methods}

\section{Participants}

136 In total, 68 participants (34 female, 34 male) participated in the experiment. Participants

137 with all air-conduction thresholds better than or equal to $20 \mathrm{~dB} \mathrm{HL}$ were defined as 138 normal hearing and served as a reference group, resulting in 23 normal hearing $(\mathrm{NH})$

139 participants. The other 45 participants were assigned to the hearing impaired (HI) group

140 and had varying degrees of hearing impairment (Figure 1). NH participants were between

14117 and 61 years (median age: 22 years, interquartile range: 27 years), and HI participants

142 were between 18 and 71 years (median age: 59 years, interquartile range: 12 years). All

143 participants took part voluntarily and signed an informed consent form. The study was

144 approved by the medical ethical committee of the University Hospitals Leuven.

145 Methodology

146 General study procedures and materials

147 Per participant, all data were obtained in one session of about one hour. The tests were

148 conducted in a quiet room. Audiograms were obtained for both ears, followed by multiple

149 DTTs in the better ear.

150 Pure tone air- and bone- conduction thresholds were measured at octave

151 frequencies from $250 \mathrm{~Hz}$ to $8 \mathrm{kHz}$ according to the 5-up 10-down Hughson-Westlake

152 method (Carhart and Jerger, 1959) with masking according to the method of Hood (Hood,

153 1960) if required. A Madsen Midimate 622 audiometer was used, connected to TDH-39

154 headphones and a B71 bone vibrator, calibrated according to ISO standards.

155 Next, 11 DTTs were performed by every participant in the better ear: one bilateral 156 (diotic) training test with 12 trials, and a test and retest of the 5 DTT conditions described 
157 below, in random order. The tests were conducted on 7 inch Google Nexus tablets,

158 connected to DD45 transducers embedded in Peltor caps, calibrated to the test noise at 80 159 dB SPL.

160 Identical instructions were given prior to each test by means of an instruction

161 screen. Participants were instructed to respond with 3 digits, with the response buttons

162 for 7 [ze:vən], 9 [ne:gən] and 0 [nyl] disabled, and could respond during stimulus

163 presentation. They completed the tests without supervision of the test leader. Regular 164 pauses were held in order to avoid fatigue.

165

166

167

168

169

170

\section{Digit Triplet Test conditions}

Five different conditions were investigated, using the speech and noise material described in more detail in the Appendix. The names of the procedures consist of a letter denoting the type of scoring ( $\mathrm{T}$ and $\mathrm{D}$ for triplet and digit scoring, respectively), the recognition probability or target point for a single digit, and an optional suffix LP for the test condition using a low-pass filtered noise, e.g. T79LP or D50. The specific parameter settings are summarized in Table 1 and Figure 2. Except for the training test with 12 trials, each test consisted of 27 triplets in speech-shaped noise, presented monaurally (to the better ear). One of 10 balanced test lists was randomly chosen, and triplets were presented in random order. For each test, the SRT was calculated by averaging the SNRs of the last 21 triplets presented, plus the SNR of a virtual (non-presented) $28^{\text {th }}$ triplet. The first six triplets were not used for the SRT calculation.

Triplet scoring procedure (T79). Procedure T79 is the baseline procedure as described in Jansen et al. (2013). Digit triplets were presented in a continuous broadband speech-shaped noise masker that is spectrally identical to the speech material used, fixed at $65 \mathrm{~dB}$ SPL. A simple up-down adaptive procedure was used, varying the speech level with fixed steps of $2 \mathrm{~dB}$ according to the identification response for a whole triplet (triplet 
182 scoring), i.e. all three digits needed to be identified correctly to decrease the SNR. The

183 first triplet was presented at an SNR of $0 \mathrm{~dB}$. Digits had been equated for intelligibility 184 in this type of noise (Jansen et al, 2013). A test with the same parameters was also used

185 for training, but was presented bilaterally with only 12 (instead of 27) trials.

186 Triplet scoring procedure with low-pass filtered noise. Procedure T79LP also

187 used triplet scoring and a simple up-down adaptive procedure. The original background 188 noise was replaced by a low-pass filtered version, and digits had been equated for 189 intelligibility in this type of noise (Appendix). To compensate for the attenuation effect 190 of the low-pass filter, a fixed noise level of $75 \mathrm{~dB}$ SPL was used. The initial step size was

191 twice as large as for the baseline procedure T79 to provide the required rapid descent to 192 reach the proximity of the SRT for NH participants within the first 6 triplets not used for 193 the SRT calculation.

194 Digit scoring procedures targeting different recognition probabilities. Procedures 195 D79, D57 and D35 again used the broadband noise of the baseline procedure T79, fixed 196 at $65 \mathrm{~dB}$ SPL. Adaptive step sizes depending on the number of correctly recognized digits 197 were used (digit scoring), parametrized to converge to recognition probabilities of 79, 57

198 and $35 \%$ (equation 2). To provide the same rate of descent as for the baseline procedure, 199 a recognition probability of 50\% was used for the first 6 trials. To allow for the correct 200 association of presented and recognized digit, feedback about the position of the 201 presented digit was provided by highlighting the corresponding response field.

\section{Statistical analysis}

203 Statistical calculations were performed using the R software environment (version 3.2.3)

204 for statistical computing and graphics (R Core Team, 2015). 
The influence of background noise type and scoring method (for procedures

206 targeting a recognition probability of 79\%), and the influence of recognition probability

207 (for digit scoring procedures) on the SRT was investigated, both for all and only $\mathrm{NH}$

208 participants. For each procedure, mean SRTs were calculated per participant by averaging

209 test- and retest-SRTs. When multiple factors were involved, the effect of each

210 independent factor was analysed with random-effects ANOVAs. Normality of individual

211 variables was checked by Shapiro-Wilk tests. For normally distributed variables, within-

212 or between-subject effects were tested with paired or unpaired Student's t-tests,

213 respectively. Otherwise, Wilcoxon signed-rank or Mann-Whitney U tests were used.

214 Test-retest errors (TRTE) were calculated to estimate the reliability of the DTT,

215 i.e. the deviation of test results from the underlying actual threshold. Individual TRTEs

216 were calculated per participant and DTT condition as the signed sample-based standard

217 deviation. Across participants, e.g. for the NH and HI groups, the TRTE was calculated

218 as the standard deviation of the individual TRTEs (Smits \& Houtgast, 2007). This

219 definition results in a TRTE estimate that has the same variability as the difference

220 between estimated and underlying actual threshold. To evaluate test-retest reliability, the

221 absolute deviation from the median was calculated as a robust measure of variability.

222 Significant differences in variability with respect to type of background noise and scoring

223 method used (for procedures targeting a recognition probability of $79 \%$ ), as well as the

224 recognition probability targeted (for digit scoring procedures) were tested with Wilcoxon

225 signed-rank tests.

SRTs were compared to the PTA calculated from the average audiometric threshold at 1, 2 and $4 \mathrm{kHz}$. Pearson correlation coefficients between PTA and SRT were calculated per procedure after averaging test- and retest-SRTs per participant. 
230 and HI participants. For the $79 \%$ recognition probability, the influence of background

231 noise and scoring method was analysed. For the digit scoring procedures, the influence

232 of the recognition probability was investigated. Significant differences for two dependent

233 correlations sharing one variable were tested with a two-tailed Williams's Test.

234 Dependent correlations with no shared variables were tested with a two-tailed Steiger

235 Test. To control the family-wise error rate for multiple comparisons, the Holm-

236 Bonferroni method was used.

237 Bootstrapping with $\mathrm{n}=1000$ iterations was used to calculate $95 \%$ confidence 238 intervals. 


\section{Speech Reception Thresholds}

241 Figure 3 shows the distribution of SRTs per procedure for $\mathrm{HI}$ and NH participants. Across

242 all participants, for procedures targeting a recognition probability of $79 \%$, mean SRTs

243 from T79LP were 9.6 dB lower (better) than from T79, and no significant difference was

244 found between D79 and T79 ( $\mathrm{p}<0.001$ and $\mathrm{p}>0.05$, respectively). For the digit scoring

245 procedures, mean SRTs from D79 were $2 \mathrm{~dB}$ higher (worse) than from D57, which were

246 in turn $2.3 \mathrm{~dB}$ higher than those of D35 $(\mathrm{p}<0.001)$.

247 For NH participants, mean SRTs from T79LP were about $13.3 \mathrm{~dB}$ lower than from

248 T79, and no significant difference was found between D79 and T79 $(\mathrm{p}<0.001$ and $\mathrm{p}>$

2490.05 , respectively). For the digit scoring procedures, mean SRTs from D79 were $1.5 \mathrm{~dB}$

250 higher than from D57, which were in turn $1.9 \mathrm{~dB}$ higher than those of D35 $(\mathrm{p}<0.001)$.

\section{Test-retest reliability}

252 Table 2 shows the TRTEs for the different procedures for NH and HI participants. Across 253 all procedures, TRTEs in HI participants were $0.19 \mathrm{~dB}$ higher than in NH participants 254 (95\% CI [0.09-0.29] $\mathrm{dB}, \mathrm{p}<0.001)$. With digit scoring procedure D79, TRTEs were 255 significantly lower by $0.19 \mathrm{~dB}$ compared to triplet scoring procedure $\mathrm{T} 79$ (95\% CI [0.02$2560.36] \mathrm{dB}, \mathrm{p}<0.05)$. No significant difference in test-retest reliability was observed for T79 257 and T79LP $(\mathrm{p}>0.05)$.

\section{SRT-PTA correlations}

259 Figure 4 shows scatterplots of the SRT versus PTA. All correlations between PTA and

260 SRT were significant when tested for all participants and only HI participants, but none 261 for only $\mathrm{NH}$ participants $(\mathrm{p}<0.001$ and $\mathrm{p}>0.05$, respectively). When comparing 
262 correlations for the different methods using a $79 \%$ recognition probability, i.e. T79,

263 T79LP and D79, no significant differences were found $(\mathrm{p}<0.05)$, both neither for all 264 participants (mean $r=0.90)$ nor for only HI participants (mean $r=0.86)$. When comparing 265 correlations for the digit scoring procedures at different recognition probabilities (D79, 266 D57 and D35,) D79 yielded better scores than D57 and D35, and D57 better than D35 267 ( $\mathrm{p}<0.05)$, both for all participants as well as for only HI participants. 


\section{Discussion}

269 This study investigated modifications of the DTT in order to improve its efficiency. The

270 use of a low-pass filtered noise as well as the use of variable adaptive step sizes according

271 to a digit scoring procedure were explored. These modifications were hypothesized to

272 either increase the spread in SRTs among participants (low-pass filtered noise) or increase

273 test-retest reliability (digit scoring), leading to improved test efficiency.

\section{Baseline procedure}

275 For the baseline procedure T79, SRTs for the NH participants were very similar to the 276 mean SRT of -11.7 dB SNR reported for the same material in Jansen et al. (2013). Also, 277 test-retest reliability was similar to values previously reported for the Flemish DTT 278 (Jansen et al, 2013; Jansen et al, 2014) and DTTs for other languages. Test-retest 279 reliability was lower in HI participants, which might have been caused by the shallower 280 slopes of their psychometric curves. Post-hoc psychometric curve fitting indeed showed 281 a decrease in slope of $1.3 \% / \mathrm{dB}$ per $\mathrm{dB}$ increase in SRT, as is also reported in the literature 282 (Smits \& Houtgast, 2005; Smits \& Houtgast, 2006; Smits \& Festen, 2011). PTA and SRT were highly positively correlated when calculated for all participants, with correlation coefficients being in line with reported values between 0.7 and 0.8 (Smits et al, 2004; Jansen et al, 2010; Watson et al, 2012; Koole et al, 2016; Potgieter et al, 2018b). The declining correlation when only including HI listeners is smaller than the 0.28 seen for

287 the French DTT in Jansen et al. (2012), which might be because of the more loose criterion for HI participants in that study. For only NH participants, the SRTs were not sufficiently sensitive to detect differences in PTAs. This was expected given the strict definition of normal hearing with all air-conduction thresholds better than or equal to 20 dB HL. 


\section{Low-pass filtered noise procedure}

293 For T79LP, SRTs for the NH participants agreed with the mean SRT of -24.6 dB SNR

294 obtained in its development experiment (Appendix). As expected, SRTs for the low-pass

295 filtered noise procedure decreased and were more variable when compared to T79, i.e.

296 SRTs were more spread out for the HI participants depending on their high frequency

297 audiometric threshold (Leensen et al, 2011; Jansen et al, 2014; Vlaming et al, 2014). No

298 significant difference in test-retest reliability was observed for T79LP and T79. This is in

299 accordance with the results of Leensen et al. (2011) for Dutch CVC words. Correlations

300 between SRTs and PTAs for both procedures were around 0.9 and did also not differ

301 between procedures, presumably because correlations for T79 were already high. These

302 results suggest that the use of a low-pass filtered noise would not result in improved test

303 efficiency, which is in contrast to findings reported by other researchers, who reported

304 increases in absolute correlations when switching from a broadband to a low-pass filtered 305 noise using English digit triplets (from 0.62 to 0.79: Vlaming et al, 2014), or Dutch (from

3060.75 to 0.91: Leensen et al, 2011), Flemish (from 0.64 to 0.79: Jansen et al, 2014) and

307 French (from 0.73 to 0.91: Jansen et al, 2014) CVC words.

308 To investigate whether the use of a low-pass filtered noise might nevertheless

309 capture additional information about hearing impairment, linear modelling was used to

310 predict T79LP-SRTs from T79-SRTs and pure tone thresholds. In a first model with only

311 the T79-SRT as predictor, T79LP-SRTs could be predicted with an $\mathrm{R}^{2}$ of $86.6 \%$

$312(\mathrm{p}<0.001)$. Using the Akaike Information Criterion (AIC), pure tone thresholds were

313 included step-wise to obtain the best fit possible with as few variables as possible. This

314 resulted in a second model that included pure tone thresholds at 0.5 and $2 \mathrm{kHz}$, which

315 significantly increased the proportion of explained variance to $92.3 \%(\mathrm{p}<0.001)$. This

316 indicates that the low-pass filtered noise procedure captured additional information about 
317 the pure tone thresholds that is not captured by T79. Most likely, the thresholds at 0.5 and

$3182 \mathrm{kHz}$ provided the necessary information on the amount and slope of high-frequency

319 hearing impairment. The remaining unexplained variance might be rather independent of

320 hearing impairment and could be dominated by random effects, such as measurement

321 error.

\section{Digit scoring procedures}

323 Digit scoring allowed for the use of different recognition probabilities, resulting in 324 different SRTs. SRTs for D79 did not differ significantly from T79. For lower recognition 325 probabilities, SRT variability decreased, similar to what has been reported by Smits \& 326 Houtgast, 2007. This can be related to the effect of hearing impairment on the parameters

327 of the psychometric curve. With increasing hearing impairment, the psychometric curve 328 is not so much shifted to higher SNRs, but actually $\mathrm{p}$ anchored as chance level is 329 approached. In this way, both midpoint (SRT) and slope are affected. Therefore, for 330 participants with different hearing impairment degrees, the variability of the upper-right 331 part of the curve will be higher than in the lower part (Smits \& Festen, 2011; Smits \& 332 Festen, 2013).

333 Digit scoring procedure D79 resulted in significantly higher test-retest reliability 334 compared to T79. This is most probably due to the increase in the number of effective 335 stimuli used for D79 psychometric curve calculations and the SNRs used for the SRT 336 calculation being distributed more evenly and closer to the recognition probability, which 337 is in agreement with the results for matrix sentence tests (Brand \& Kollmeier, 2002). On 338 the other hand, SRT-PTA correlations for procedures D79 and T79 did not differ 339 significantly, indicating improved test efficiency. 
341 have been caused by the larger ratio of accidentally correct answers relative to the 342 expected recognition probabilities. This is in agreement with simulations done by Smits

$343 \&$ Festen (2011). In their study, the most reliable recognition probability for shallow

344 slopes of the psychometric curve was found to be around $80 \%$. Also, correlations between

345 PTAs and SRTs for lower recognition probabilities decreased, which might have been

346 partially caused by the higher measurement errors.

\section{$347 \quad$ Implications and future research perspectives}

348 Altogether, using a recognition probability of $79 \%$ seems valid, and a better choice than 349 using lower probabilities. The use of variable adaptive step sizes according to a digit scoring method improves test efficiency due to an increased test reliability, and, therefore, yields a more efficient test than does triplet scoring with a fixed step size. Relative to the reliability of the currently used Flemish DTT, test-retest reliability increased by $0.2 \mathrm{~dB}$.

353 Conveniently, SRTs remain unaltered avoiding the need to establish new normative values or pass/fail-criteria. However, its performance should be re-evaluated in a larger study sample for further validation, especially in terms of its sensitivity and specificity to detect hearing impairment, e.g. in the context of occupational or school health hearing screening.

Also, with respect to test efficiency, so far, test duration has not been taken into account. Nevertheless, hearing screening tests are best kept as short as possible to have optimal cost-efficiency. Whereas test duration can simply be reduced by presenting fewer triplets or trials, such a reduction will result in a loss of reliability. Under the assumption

362 of independence of the individual responses, test reliability is expected to decrease with

363 the number of trials $n$ used for the SRT calculation, i.e. TRTE $\sim \sqrt{n}^{-1}$. However, given 
364 the increased test-retest reliability using digit scoring, a similar TRTE as for T79 would

365 already be reached after 17 triplets for D79, corresponding to a reduction in triplets of $36637 \%$. Such a test would then be as efficient as the currently used DTT with triplet scoring 367 and 27 trials, provided that its correlation with the PTA remains unchanged. To simulate 368 a shorter test with a total number of 17 triplets, the SRTs for D79 were recalculated from 369 the SNRs of triplets 12 to 23 . This resulted in a TRTE of $0.86 \mathrm{~dB}$ and an SRT-PTA correlation of 0.92 . Such a shorter test should be evaluated in future research regarding its reliability and validity, especially in the light of possible attention effects because of the changed number of triplets (Witton et al, 2017). Indisputably, screening programs using the DTT might benefit from a shorter test, especially when targeting children who

374 have limited attention spans. In Flanders, where the DTT is used for school-age hearing screening (Denys et al, 2018; Guérin et al, 2018), test durations of about 6 to 7 minutes have been reported for $3^{\text {rd }}$ grade elementary and $5^{\text {th }}$ grade secondary school children, which can be considered rather long for screening. More importantly, attention loss during the test, resulting in poor SRTs, might add to the proportion of unjustified referrals. One way to minimize this, could be the use of a shorter (and hence more efficient) test.

380 Recently, a fixed SNR procedure was introduced, in which the average number of presentations needed was only 30 to $50 \%$ of the number of presentations used for an adaptive procedure, whilst having equal pass and refer rates (Smits, 2017). Importantly, especially when using faster procedures that use fewer triplets,

384 optimal convergence to the SRT within the first 6 trials not used for its calculation, should 385 be obtained. The initial recognition probability for the digit scoring procedures evaluated in this study was set to $50 \%$, as digit scoring for the $79 \%$ recognition probability would

387 have led to a slow descent (the maximum step size $=0.83 \mathrm{~dB}$ ). The speed of convergence 388 for all procedures was investigated (Figure 5). Depending on the procedure, the SNR of 
389 a certain number of triplets differed significantly from the SRT $(\mathrm{p}<0.05)$. For the triplet

390 scoring procedures T79 and T79LP, convergence was achieved within the initial 6 triplets

391 allocated for descent. For the digit scoring procedures, only D57 converged properly, with

392 significant differences from the SRT up to the $11^{\text {th }}$ and $10^{\text {th }}$ triplet for procedures D79 and

393 D35, respectively. While procedure D35 undershot the SRT at the $7^{\text {th }}$ triplet with about

$3942.4 \mathrm{~dB}$ on average, procedure D79 exhibited a mean overshoot of about $1.7 \mathrm{~dB}$ that took

395 several triplets to recover. This was worse for HI participants. For the $79 \%$ recognition

396 probability, a procedure which would use triplet scoring during the initial descent (the

397 first 5 triplets) and digit scoring for the remaining triplets might be a possible solution.

398 Such a procedure should be evaluated in future studies using the DTT with digit scoring,

399 albeit with 27 or 17 trials. 
401 This study aimed to improve the efficiency of the Flemish DTT. The use of a low-pass

402 filtered noise did not result in an improved SRT-PTA correlation or increased reliability.

403 Using digit scoring with variable adaptive step sizes parametrized to target a recognition

404 probability of $79 \%$ improved test-retest reliability. This procedure should be further 405 evaluated with respect to its sensitivity and specificity to detect hearing impairment, the 406 more because it offers opportunities to reduce the number of trials (and hence test 407 duration), which would be beneficial for large-scale screening programs in which the test 408 is already being used. Targeting lower recognition probabilities decreased test-retest 409 reliability, as well as correlations with the PTA and should not be used. 
411 In this appendix, we describe the optimization of the DTT speech material for use in low412 pass filtered noise, and its evaluation in NH participants. The speech and noise material of the Flemish DT as described in Jansen et al.

414 (2013) was used. This material consists of 10 balanced lists of 27 digit triplets each, with 415 digits equated for intelligibility in broadband speech-shaped noise. Only the monosyllabic 416 digits 1 [e:n], 2 [twe:], 3 [dri:], 4 [vi:r], 5 [veif], 6 [zes] and 8 [axt] are used, which were 417 recorded by a professional female native Flemish speaker, and concatenated into digit 418 triplets. Per list, every digit occurs 3 to 4 times at every position in the triplet. Digits have 419 a common length of $601 \mathrm{~ms}$ and are presented with gaps of $200 \mathrm{~ms}$ between them. The 420 original broadband speech-shaped background noise was replaced by a low-pass filtered 421 version, as described in Jansen et al. (2014). It consists of the sum of the speech-shaped noise, low-pass filtered at $1.4 \mathrm{kHz}$ with a roll-off slope of more than $100 \mathrm{~dB}$ per octave, and the original speech-shaped noise attenuated by $15 \mathrm{~dB}$. The noise starts $1 \mathrm{~s}$ before and stopped $500 \mathrm{~ms}$ after each triplet, and has a $50 \mathrm{~ms}$ fade using a Hann window.

Stimuli were played back using APEX 3 software (Francart et al, 2008; Francart et al, 2017), installed on a Dell Latitude D510 laptop, routed through an external Fireface UCX soundcard connected to Sennheiser HDA-200 headphones and presented monaurally to the best ear. Responses were typed in by the participants using a numerical touch pad connected to the laptop.

Six NH adults (thresholds at all octave frequencies $\leq 15 \mathrm{~dB}$ HL, confirmed by standard pure tone audiometry) participated in the optimization experiment. Following a training list at $-18 \mathrm{~dB}$ SNR, test lists were presented at several fixed SNRs, ranging from $433-22$ to $-30 \mathrm{~dB}$ SNR in steps of $2 \mathrm{~dB}$ relative to a fixed noise level of $65 \mathrm{~dB}$ SPL. Per SNR, 4342 test lists were presented, and test lists used were balanced among participants. 
435 Responses were scored at the level of individual digits. Per digit, a psychometric curve

436 was fitted using equation (3). The level of individual digits was adjusted by the difference

437 in SRT for the individual digit and the average SRT for all digits, to obtain equated

438 intelligibility and a homogenous test material with a steeply sloped psychometric curve.

439 This was done to obtain the highest possible test reliability. Level corrections performed

440 ranged from -1.6 to $1.2 \mathrm{~dB}$, but were generally $<1 \mathrm{~dB}$.

441 Twelve NH participants participated in the evaluation experiment. Here, reference 442 values were determined for the test at adaptively varying as well as at fixed SNRs. Per 443 participant, first, 11 tests lists (including 1 list for training) were performed adaptively 444 with a start-SNR of $-18 \mathrm{~dB}$ and a fixed step size of $2 \mathrm{~dB}$, followed by $4 \times 3$ test lists at 445 fixed SNRs of $-22,-23.5,-25$ and $-26.5 \mathrm{~dB}$, relative to a noise level of $75 \mathrm{~dB}$ SPL. Test 446 lists were again presented with balanced order. Responses were now scored at the level 447 of triplets. Per participant, a psychometric curve was fitted to the data obtained at fixed 448 SNRs, again using equation (3). This resulted in a reference curve with a slope of $44921.5 \% / \mathrm{dB}$ (as opposed to $17.5 \% / \mathrm{dB}$ prior to optimization) and a reference-SRT $\pm 2 \mathrm{SD}$ of $450-25.1 \pm 1.6 \mathrm{~dB}$ SNR. When fitted per list, SRTs fell within a range of $1 \mathrm{~dB}$ of the 451 reference-SRT. For the adaptive procedure, SRTs were determined by averaging the 452 SNRs of the last 21 presented triplets and a $28^{\text {th }}$ non-presented triplet for which the SNR 453 was calculated from the response for the lastly presented triplet. This resulted in a 454 reference-value $\pm 2 \mathrm{SD}$ of $-24.6 \pm 1.4 \mathrm{~dB}$ SNR. The reliability of the SRT-estimate was 455 calculated by taking the root mean square of the SDs across repeated tests per participant, 456 and was $0.7 \mathrm{~dB}$. 
Akeroyd, M.A., Arlinger, S., Bentler, R.A., Boothroyd, A., Dillier, N., et al, 2015. International Collegium of Rehabilitative Audiology (ICRA) recommendations for the construction of multilingual speech tests. International journal of audiology, 54(sup2), p.17-22.

Brand, T., Kollmeier, B., 2002. Efficient adaptive procedures for threshold and concurrent slope estimates for psychophysics and speech intelligibility tests. The Journal of the Acoustical Society of America, 111(6), p.2801-2810.

Denys, S., Hofmann, M., Luts, H., Guerin, C., Keymeulen, A., et al, 2018. School-Age Hearing Screening Based on Speech-in-Noise Perception Using the Digit Triplet Test. Ear and hearing, 39(6), p.1104-1115.

Folmer, R.L., Vachhani, J., Mcmillan, G.P., Kidd, G.R., Feeney, M.P., 2017. Validation of a Computer-Administered Version of the Digits-in-Noise Test for Hearing Screening in the United States. Journal of the American Academy of Audiology, 28(2), p.161-169.

Francart, T., Hofmann, M., Vanthornhout, J., Van Deun, L., van Wieringen, A., et al, 2017. APEX/SPIN: a free test platform to measure speech intelligibility. International journal of audiology, 56(2), p.137-143.

Francart, T., van Wieringen, A., Wouters, J., 2008. APEX 3: a multi-purpose test platform for auditory psychophysical experiments. Journal of Neuroscience Methods, 172, p.283-293.

Guérin, C., Van Hoeck, K., Denys, S., van Wieringen, A., Wouters, J., et al, 2018. Systematische opsporing van lawaaischade bij jongeren. JGZ Tijdschrift voor Jeugdgezondheidszorg, 50(5-6), p.132-137.

Jansen, S., Luts, H., Dejonckere, P., van Wieringen, A., Wouters, J., 2013. Efficient hearing screening in noise-exposed listeners using the digit triplet test. Ear and hearing, 34(6), p.773-778.

Jansen, S., Luts, H., Dejonckere, P., van Wieringen, A., Wouters, J., 2014. Exploring the sensitivity of speech-in-noise tests for noise-induced hearing loss. International journal of audiology, 53(3), p.199-205.

Jansen, S., Luts, H., Wagener, K.C., Frachet, B., Wouters, J., 2010. The French digit triplet test: a hearing screening tool for speech intelligibility in noise. International journal of audiology, 49(5), p.378-387.

Jansen, S., Luts, H., Wagener, K.C., Kollmeier, B., Del Rio, M., et al, 2012. Comparison of three types of French speech-in-noise tests: a multi-center study. International journal of audiology, 51(3), p.164-173. 
Koole, A., Nagtegaal, A.P., Homans, N.C., Hofman, A., Baatenburg de Jong, R.J., et al, 2016. Using the Digits-In-Noise Test to Estimate Age-Related Hearing Loss. Ear and hearing, 37, p.508-513.

Leensen, M.C.J., Dreschler, W. a, 2013. The applicability of a speech-in-noise screening test in occupational hearing conservation. International journal of audiology, 52(7), p.455-65.

Leensen, M.C.J., de Laat, J. a P.M., Snik, A.F.M., Dreschler, W. a, 2011. Speech-innoise screening tests by internet, part 2: improving test sensitivity for noiseinduced hearing loss. International journal of audiology, 50(11), p.835-48.

Potgieter, J.-M., Swanepoel, D.W., Myburgh, H.C., Hopper, T.C., Smits, C., 2015. Development and validation of a smartphone-based digits-in-noise hearing test in South African English. International journal of audiology, 55(7), p.405-411.

Potgieter, J.-M., Swanepoel, D.W., Myburgh, H.C., Smits, C., 2018a. The South African English Smartphone Digits-in-Noise Hearing Test: Effect of Age, Hearing Loss, and Speaking Competence. Ear and hearing, 39(4), p.656-663.

Potgieter, J.-M., Swanepoel, D.W., Smits, C., 2018b. Evaluating a smartphone digits-innoise test as part of the audiometric test battery. The South African journal of communication disorders $=$ Die Suid-Afrikaanse tydskrif vir Kommunikasieafwykings, 65(1), p.e1-e6.

Pronk, M., Deeg, D.J.H., Festen, J.M., Twisk, J.W., Smits, C., et al, 2013. Decline in older persons' ability to recognize speech in noise: the influence of demographic, health-related, environmental, and cognitive factors. Ear and hearing, 34(6), p.722-732.

Pronk, M., Deeg, D.J.H., Smits, C., Twisk, J.W., van Tilburg, T.G., et al, 2014. Hearing Loss in Older Persons: Does the Rate of Decline Affect Psychosocial Health? Journal of aging and health, 26(5), p.703-723.

Rashid, M.S., Leensen, M.C.J., Dreschler, W.A., 2016. Application of the online hearing screening test "Earcheck": Speech intelligibility in noise in teenagers and young adults. Noise and Health, 18, p.312-318.

Sheikh Rashid, M., Leensen, M.C., de Laat, J.A., Dreschler, W.A., 2017. Crosssectional evaluation of an internet-based hearing screening test in an occupational setting. Scandinavian journal of work, environment \& health, 43(3), p.279-286.

Smits, C., 2017. Improving the Efficiency of Speech-In-Noise Hearing Screening Tests. Ear and hearing, 38(6), p.e385-e388.

Smits, C., Festen, J.M., 2013. The interpretation of speech reception threshold data in normal-hearing and hearing-impaired listeners: II. Fluctuating noise. The Journal of the Acoustical Society of America, 133(5), p.3004-15. 
Smits, C., Festen, J.M., 2011. The interpretation of speech reception threshold data in normal-hearing and hearing-impaired listeners: steady-state noise. The Journal of the Acoustical Society of America, 130(5), p.2987-98.

Smits, C., Houtgast, T., 2006. Measurements and calculations on the simple up-down adaptive procedure for speech-in-noise tests. The Journal of the Acoustical Society of America, 120(3), p.1608-21.

Smits, C., Houtgast, T., 2007. Recognition of digits in different types of noise by normal-hearing and hearing-impaired listeners. International journal of audiology, 46(3), p.134-44.

Smits, C., Houtgast, T., 2005. Results from the Dutch speech-in-noise screening test by telephone. Ear and hearing, 26(1), p.89-95.

Smits, C., Kapteyn, T.S., Houtgast, T., 2004. Development and validation of an automatic speech-in-noise screening test by telephone. International journal of audiology, 43(1), p.15-28.

Smits, C., Merkus, P., Houtgast, T., 2006. How we do it: The Dutch functional hearingscreening tests by telephone and internet. Clinical otolaryngology, 31(5), p.436440.

De Sousa, K.C., Swanepoel, D.W., Moore, D.R., Smits, C., 2018. A Smartphone National Hearing Test: Performance and Characteristics of Users. American journal of audiology, 27(3S), p.448-454.

Stam, M., Smit, J.H., Twisk, J.W.R., Lemke, U., Smits, C., et al, 2016. Change in Psychosocial Health Status Over 5 Years in Relation to Adults' Hearing Ability in Noise. Ear and hearing, 37(6), p.680-689.

Stam, M., Smits, C., Twisk, J.W.R., Lemke, U., Festen, J.M., et al, 2015. Deterioration of Speech Recognition Ability Over a Period of 5 Years in Adults Ages 18 to 70 Years : Results of the Dutch Online Speech-in-Noise Test. , p.129-137.

Vercammen, C., Goossens, T., Wouters, J., van Wieringen, A., 2018. Digit Triplet Test Hearing Screening With Broadband and Low-Pass Filtered Noise in a MiddleAged Population. Ear and hearing, 39(4), p.825-828.

Vlaming, M.S.M.G., MacKinnon, R.C., Jansen, M., Moore, D.R., 2014. Automated Screening for High-Frequency Hearing Loss. Ear and hearing, p.1-13.

Watson, C.S., Kidd, G.R., Miller, J.D., Smits, C., Humes, L.E., 2012. Telephone screening tests for functionally impaired hearing: current use in seven countries and development of a US version. Journal of the American Academy of Audiology, 23(10), p.757-767.

Willberg, T., Buschermohle, M., Sivonen, V., Aarnisalo, A.A., Lopponen, H., et al, 2016. The development and evaluation of the Finnish digit triplet test. Acta otolaryngologica, 136(10), p.1035-1040. 
568 Witton, C., Talcott, J.B., Henning, G.B., 2017. Psychophysical measurements in

$569 \quad$ children : challenges, pitfalls, and considerations. PeerJ, 5, p.e3231.

570 Zokoll, M. a, Wagener, K.C., Brand, T., Buschermöhle, M., Kollmeier, B., 2012. Internationally comparable screening tests for listening in noise in several European languages: the German digit triplet test as an optimization prototype. International journal of audiology, 51(9), p.697-707.

Zokoll, M.A., Hochmuth, S., Warzybok, A., Wagener, K.C., Buschermohle, M., et al, 2013. Speech-in-noise tests for multilingual hearing screening and diagnostics1. American journal of audiology, 22(1), p.175-178. 


\section{Figures and Tables}

579 Figure 1. Distribution of the pure tone thresholds (in dB HL) per frequency (in $\mathrm{Hz}$ ) for

580 hearing impaired (HI, left panel) and normal hearing ( $\mathrm{HH}$, right panel) participants. Each

581 black line represents a different percentile.

582 Figure 2. Step sizes (in $\mathrm{dB}$ ) according to the number of correctly recognized digits per triplet for the different Digit Triplet Test procedures for the triplets assigned to the initial descent (upper row) and for the remaining triplets (lower row).

Figure 3. Distribution of speech reception thresholds (SRT, in dB SNR) for the different

586 Digit Triplet Test procedures for hearing impaired (HI, left panel) and normal hearing

587 (NH, right panel) participants.

588 Figure 4. Scatterplots of the speech reception thresholds (SRT, in dB SNR) versus pure 589 tone averages (PTA, i.e. averaged hearing threshold over frequencies $1-4 \mathrm{kHz}$, in $\mathrm{dB} \mathrm{HL}$ )

590 for the different Digit Triplet Test procedures for normal hearing (green dots) and hearing

591 impaired (orange dots) participants. Numerical information on the graphs represent 592 correlation coefficients ( $r$ ) for all (in black) and only hearing impaired (in orange) 593 participants, with $95 \%$ confidence intervals, resulting from basic bootstrapping with $594 \mathrm{n}=1000$ iterations. Dotted lines represent linear regression fits.

595 Figure 5. Convergence plots for the different Digit Triplet Test procedures for hearing 596 impaired (HI, upper row) and normal hearing (NH, lower row) participants. Red boxes 597 indicate triplets at which the SNR is significantly different from the speech reception 598 threshold (SRT), which lies at $0 \mathrm{~dB}$ signal-to-noise ratio (SNR). The vertical black dotted 599 lines divide each plot in descending (or convergence) trials and trials used for the 600 calculation of the SRT.

601 Table 1. Parameter settings for the different Digit Triplet Test procedures used.

602 Table 2. Test-retest errors for the different Digit Triplet Test procedures used for hearing 603 impaired (HI) and normal hearing $(\mathrm{NH})$ participants. Values between brackets indicate $60495 \%$ confidence intervals, resulting from basic bootstrapping with $n=1000$ iterations. 


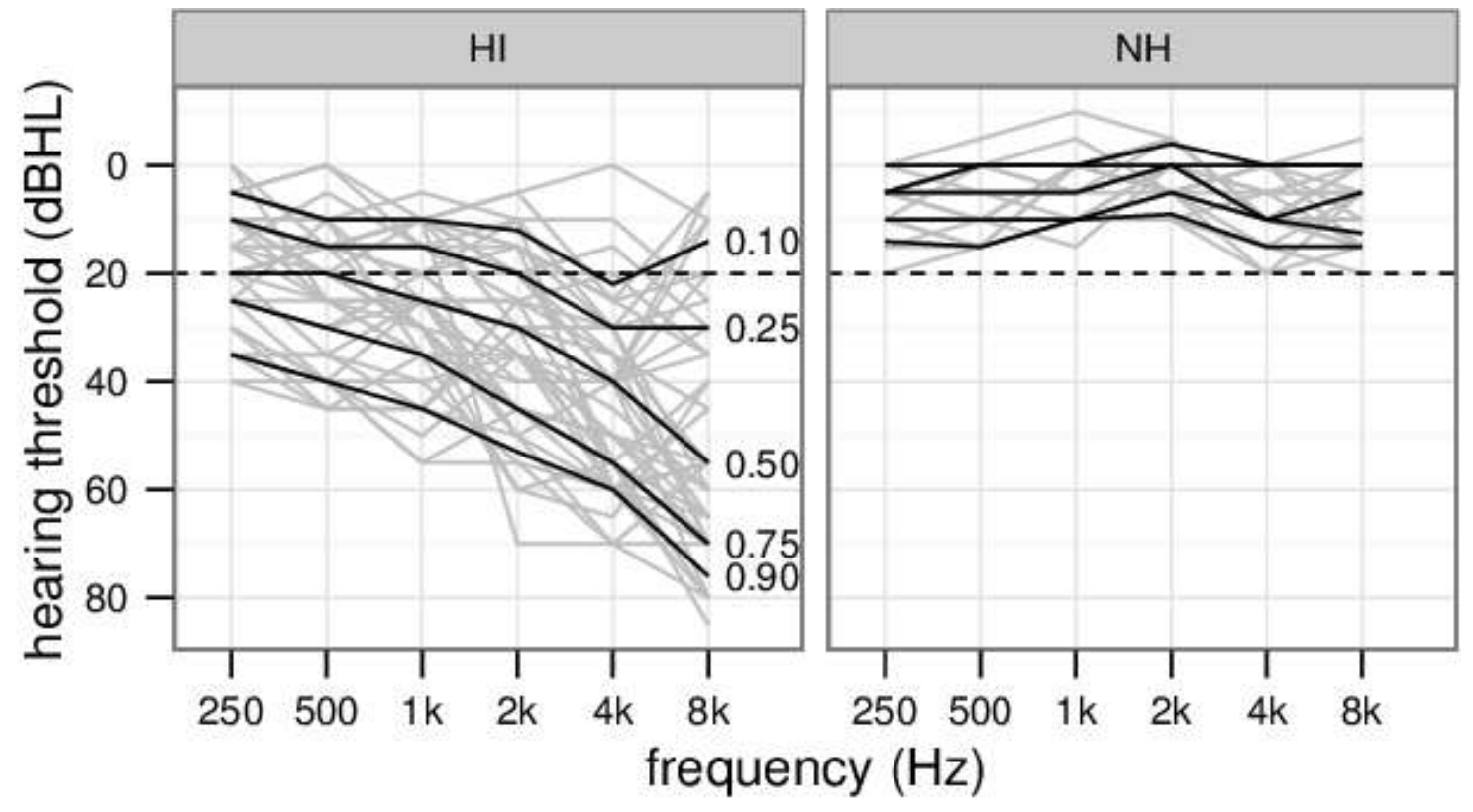

605

Figure 1 

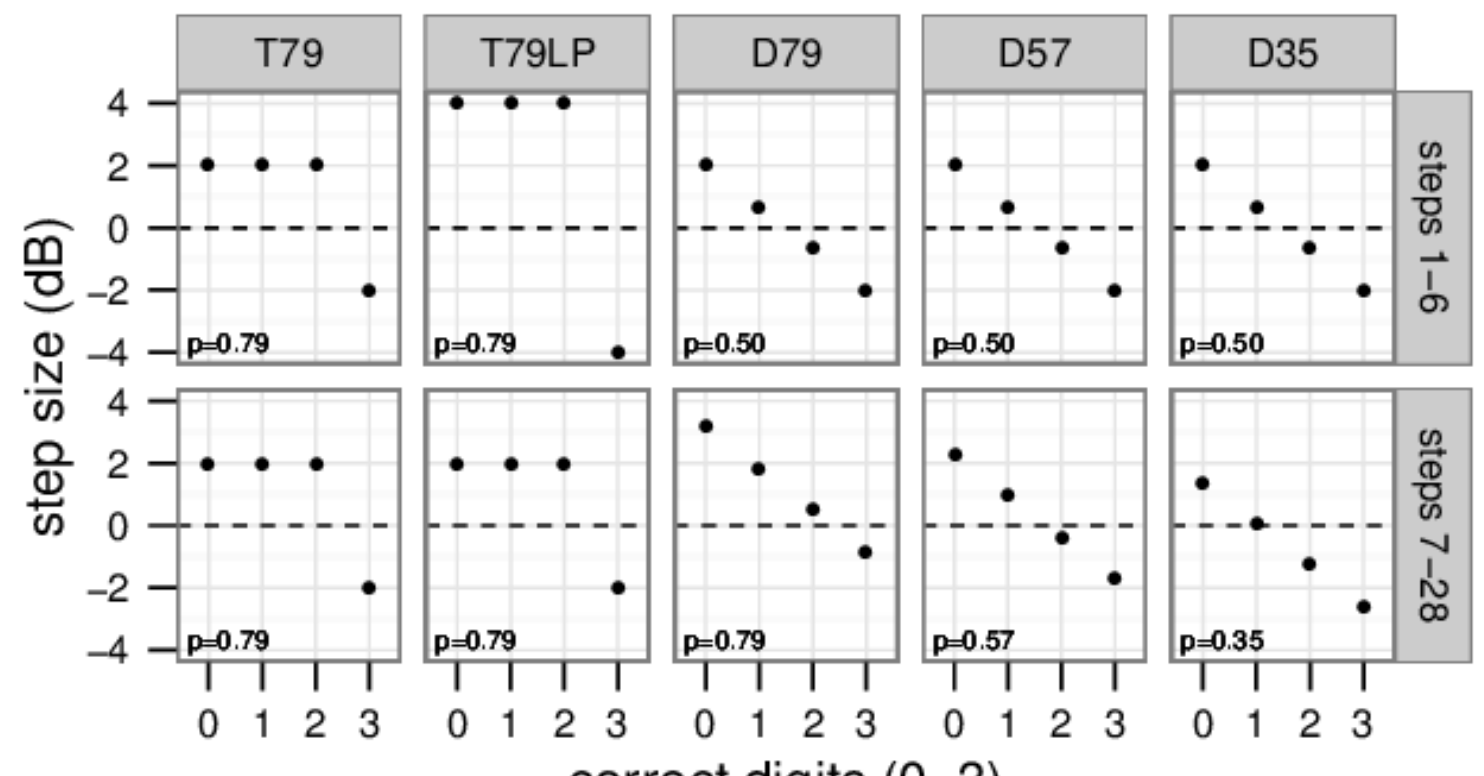

607 correct digits $(0 . .3)$

Figure 2 


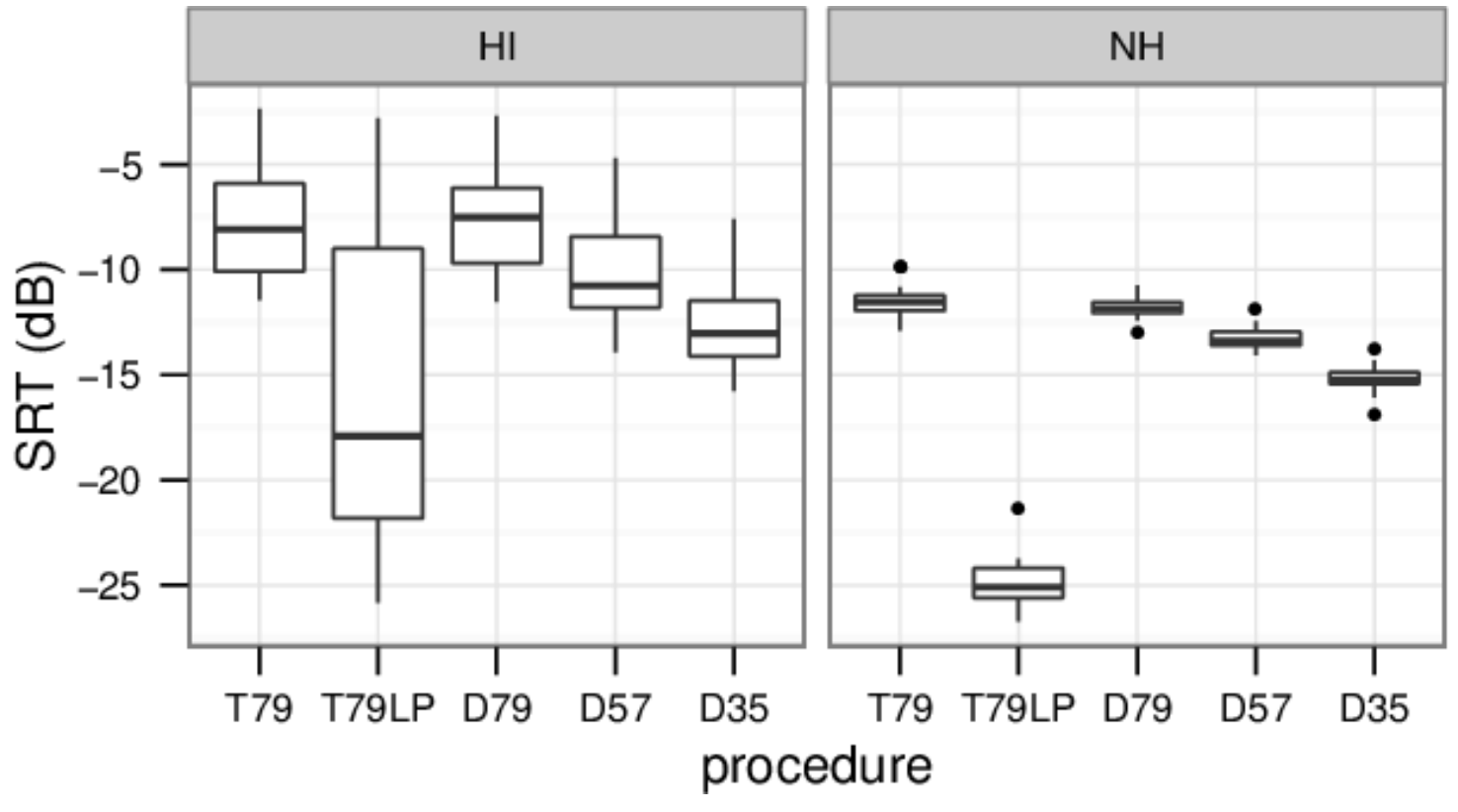

609

610

Figure 3 


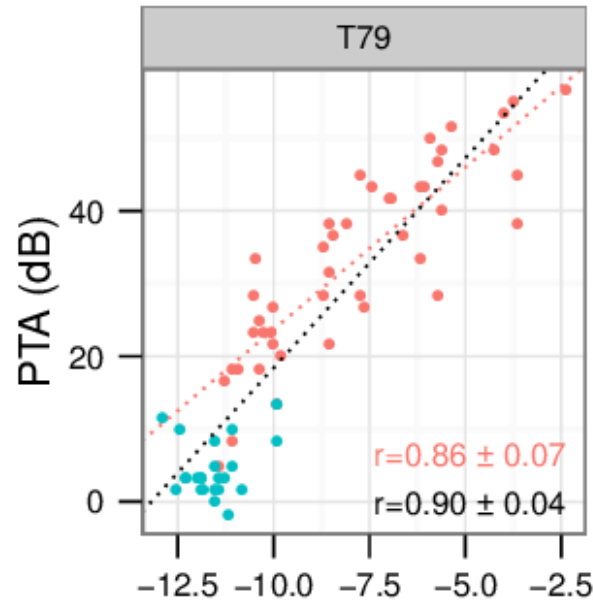

611

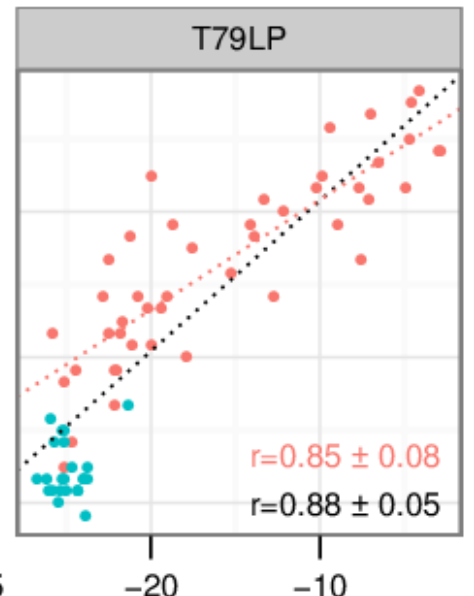

$\begin{array}{cccc}1 & 1 & 1 & 1\end{array}$ SRT (dBSPL)

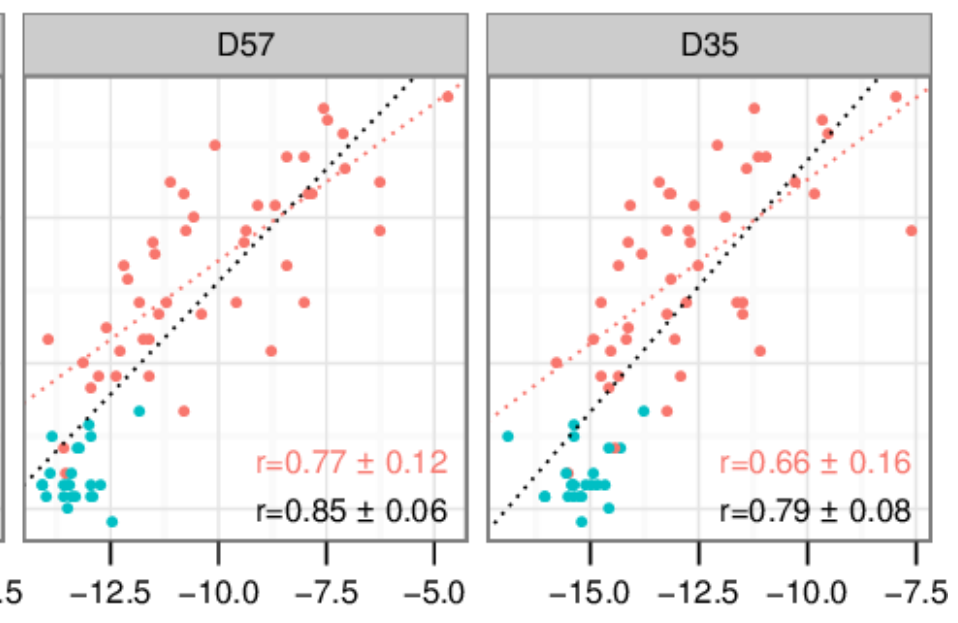

Figure 4 

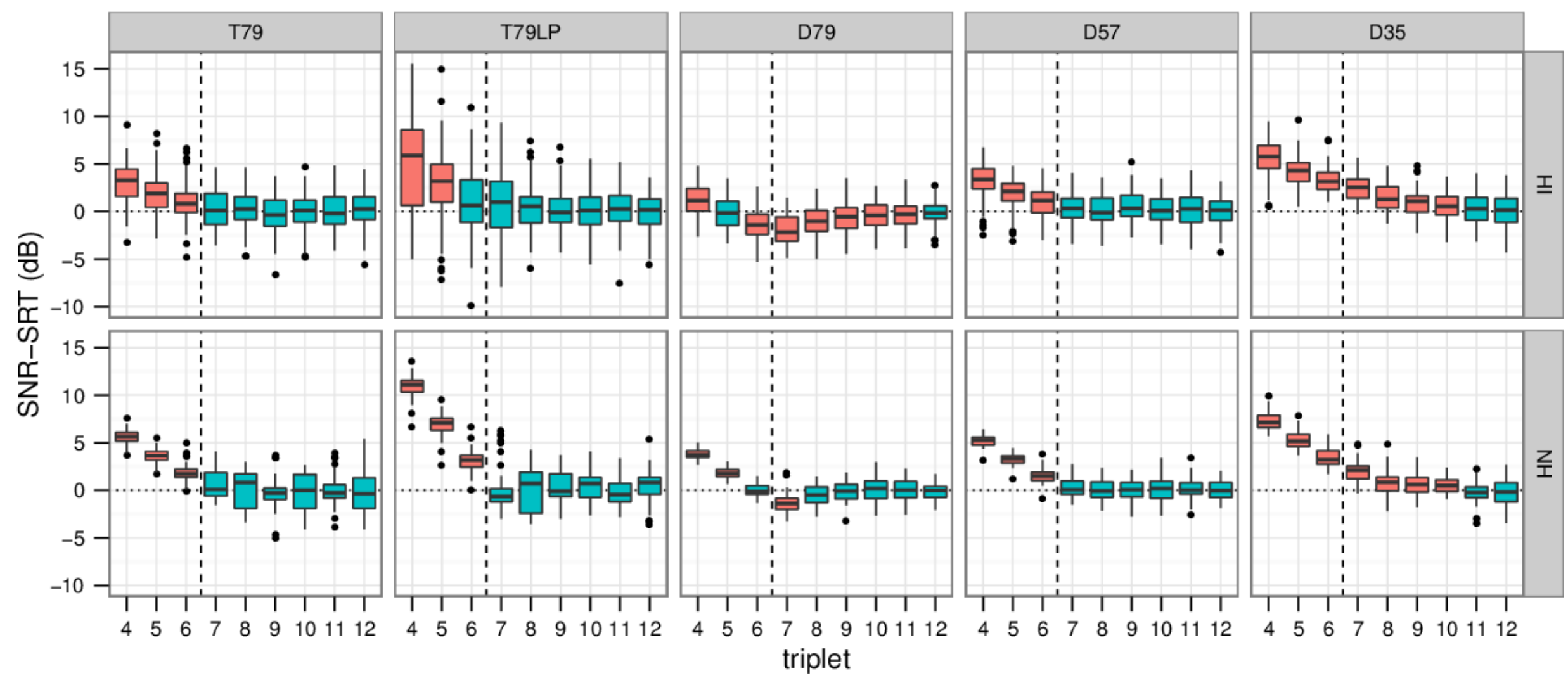

Figure 5 
Table 1

\begin{tabular}{|c|c|c|c|c|c|c|c|}
\hline Procedure & Scoring & Masker & $\begin{array}{c}\text { Masker level } \\
\text { (dB SPL) }\end{array}$ & Feedback & $\begin{array}{c}\text { Start-SNR } \\
\text { (dB) }\end{array}$ & $\begin{array}{l}p_{1-6} \\
(\%)\end{array}$ & $\begin{array}{l}p_{7-28} \\
(\%)\end{array}$ \\
\hline T79 & Triplet & Broadband & 65 & No & 0 & 79 & 79 \\
\hline T79LP & Triplet & Low-pass & 75 & No & -2 & 79 & 79 \\
\hline D79 & Digit & Broadband & 65 & Yes & -2 & 50 & 79 \\
\hline D57 & Digit & Broadband & 65 & Yes & -2 & 50 & 57 \\
\hline D35 & Digit & Broadband & 65 & Yes & -2 & 50 & 35 \\
\hline
\end{tabular}


Table 2

\begin{tabular}{ccc}
\hline Procedure & HI & NH \\
\hline T79 & $1.02[0.85-1.22]$ & $0.62[0.45-0.82]$ \\
T79LP & $0.96[0.76-1.20]$ & $0.63[0.47-0.83]$ \\
D79 & $0.76[0.61-0.93]$ & $0.45[0.32-0.58]$ \\
D57 & $0.72[0.57-0.89]$ & $0.51[0.42-0.64]$ \\
D35 & $0.87[0.69-1.08]$ & $0.76[0.54-1.02]$ \\
\hline
\end{tabular}

618 